\title{
The downregulation of miR-144 is associated with the growth and invasion of osteosarcoma cells through the regulation of TAGLN expression
}

\author{
MINGDONG ZHAO*, JIANSHU HUANG*, KEKE GUI, MIN XIONG, GUOPING CAI, JI XU, \\ KUN WANG, DECHANG LIU, XINCHAO ZHANG and WANGPING YIN \\ Department of Orthopaedics, Jinshan Hospital, Fudan University, Shanghai 200540, P.R. China
}

Received May 4, 2014; Accepted September 19, 2014

DOI: $10.3892 /$ ijmm.2014.1963

\begin{abstract}
Alterations in the expression of microRNAs (miRNAs or miRS) have been implicated in the pathogenesis of the majority of human malignancies, and the dysregulation of microRNA-144 (miR-144) has been associated with several diseases. However, the potential involvement of miR-144 in osteosarcoma, a common malignant bone tumor in children and adolescents with a high risk of relapse and metastasis, has not yet been fully investigated. In the present study, we examined the expression and roles of miRNAs in osteosarcoma as potential diagnostic markers and therapeutic targets, and we focused on miR-144 due to its known involvement in osteogenesis. We demonstrate that miR-144 is downregulated in osteosarcoma cell lines and primary human osteosarcoma tissue samples and that its ectopic expression inhibits osteosarcoma cell proliferation and invasion. We identified TAGLN as a downstream target of miR-144 and demonstrated that its expression is upregulated in osteosarcoma cell lines and tumor tissue and is inversely correlated with miR-144 expression. Our results indicate that miR-144 may regulate osteosarcoma cell proliferation and invasion by downregulating its target gene, TAGLN, suggesting that miR-144 may be a potential therapeutic target for the treatment of osteosarcoma.
\end{abstract}

\section{Introduction}

Osteosarcoma is the most common human primary malignant bone tumor in children and adolescents (1). It is associated with a high risk of local relapse or distant metastasis even after curative resection of the primary tumor and intensive chemotherapy. The 5-year survival rate for patients with osteosarcoma has

Correspondence to: Dr Wangping Yin, Department of Orthopaedics, Jinshan Hospital, Fudan University, 1508 Longhang Road, Shanghai 200540, P.R. China

E-mail:mdzhaokxy@126.com

*Contributed equally

Key words: osteosarcoma, miR-144, transgelin, growth and invasion improved significantly over the past few decades, reaching rates of approximately $60-70 \%$ since the introduction of combination chemotherapy (2). Although treatment with a combination of chemotherapy and aggressive surgical resection has markedly improved the prognosis of patients with osteosarcoma, approximately $80 \%$ of patients develop metastatic disease following surgery. Therefore, the identification of metastasis-associated molecules and the elucidation of the mechanisms contributing to invasion in osteosarcoma are essential.

microRNAs (miRNAs or miRs) are short, non-coding RNAs that regulate mRNA stability and protein translation by binding to their target mRNAs (3). Increasing evidence suggests that miRNAs play a role in the regulation of diverse biological processes (4). Since their initial identification, approximately 1,000 miRNA sequences have been identified in mammals (5). In humans, miRNAs regulate up to $30 \%$ of human genes representing the majority of genetic pathways (6). miRNAs are often deregulated in human malignancies and they have been implicated in the regulation of a number of cellular processes, including proliferation, differentiation, apoptosis and metastasis (7). The aberrant expression of miRNAs occurs in many types of cancer (8-10). miRNAs have been characterized as oncogenes, tumor suppressors or as components of regulatory pathways critical for tumorigenesis, supporting an important role for miRNAs in tumorigenesis and metastasis. However, the evaluation of deregulated miRNAs and their roles in cancer development, particularly in osteosarcoma, is an ongoing process (11).

The role of miRNAs in the development of osteosarcoma has attracted increasing attention. Certain miRNAs, including miR-143, miR-133a and miR-376c play a role in the initiation and progression of osteosarcoma and modulate the biological properties of cancer cells (12-14). Recently, the miR-144/ miR-451 cluster was found to be downregulated in osteosarcoma cells (15); however, its potential role in osteosarcoma carcinogenesis and progression remains unknown.

In the present study, we investigated the differential expression of miR-144 in osteosarcoma cells and in clinically resected human osteosarcoma tissue samples. We demonstrate that miR-144 is downregulated in osteosarcoma cells and tissue, identifying TAGLN as a target gene for miR-144. We examined the correlation between the deregulation of miR-144 
and the expression of TAGLN in osteosarcoma to elucidate their role in the growth and invasion of osteosarcoma cells. Our data indicate that miR-144 may regulate osteosarcoma cell proliferation and invasion by downregulating TAGLN, suggesting that miR-144 is a potential therapeutic target for the treatment of osteosarcoma.

\section{Materials and methods}

Materials. A total of 60 pairs of cancer tissue and corresponding normal tissue samples were collected from patients with primary osteosarcoma who received surgery between 2009 and 2011 at Jinshan Hospital, Fudan University School of Medicine (Shanghai, China). Surgically removed tissues were snap frozen in liquid nitrogen overnight and kept at $-80^{\circ} \mathrm{C}$ before use. Each specimen was verified histopathologically and scored according to the TNM staging system. The study protocol was approved by the Ethics Committee of Fudan University, Shanghai, China. All samples were collected after obtaining informed consent from all patients.

RNA extraction and reverse transcription-quantitative $P C R$ $(R T-q P C R)$. Total RNA was isolated from the osteosarcoma cell lines and tumor tissue samples using the miRNeasy kit (Qiagen, Valencia, CA, USA). To detect miRNA expression, RT-qPCR was performed using the SYBR RT-PCR kit (Takara, Shiga, Japan). The primers for TAGLN were as follows: 5'-AATGGCG TGATTCTGAGCAA-3' (forward) and 5'-CGATGCCTGCTG GAGCCGTCTA-3' (reverse). The relative expression level of TAGLN was normalized to the internal control, $\beta$-actin. Stemloop RT primers for miR-144 were as follows: 5'-GCTGGGA TATCATCATATACTG-3' (forward) and 5'-CGGACTAGTA CATCATCTATACTG-3' (reverse), as previously described (16). The primers used for quantitative PCR were as follows: 5'-ACACTCCAGCTGGGTCATGTA GTAGATA-3' (forward) and 5'-CTCAACTGGTGTCGTGGAGTCGGCAATTCAG TTGAGATGTCATA-3' (reverse). The relative expression level of miR-144 was normalized to that of the internal control U6 by using the $2^{-\Delta \Delta \mathrm{Ct}}$ cycle threshold method, as previously described (17).

Cell culture and transfection. The human normal osteoblastic cell line, hFOB1.19, and the human osteosarcoma cell lines, MG63, U2OS and HOS, were obtained from the American Type Culture Collection (ATCC, Manassas, VA, USA) and cultured in Dulbecco's modified Eagle's medium (DMEM) (Invitrogen, Carlsbad, CA, USA) supplemented with heat-inactivated $10 \%$ fetal bovine serum (FBS) at $37^{\circ} \mathrm{C}$ in a humidified incubator with $5 \% \mathrm{CO}_{2}$.

The procedure used for miR-144 eukaryotic expression vector construction was as previously described (7). The following primers were used for PCR amplification of the pri-miR-144 and native flanking sequence: 5'-GGATCCCAC AGTGCTTTTCAAGCCATG-3' and 5'-AAGCTTAGTGCC CTGGCAGTCAGTAGG-3'. The amplified fragments were then cloned into the pcDNA3.1 vector (Invitrogen) and verified by sequencing.

Transfection was performed using Lipofectamine 2000 transfection reagent (Invitrogen) according to the manufacturer's instructions. For establishing stable transfectants, the
miR-144 mimics or negative control (NC) RNA-tansfected HOS and U2OS cells were selected for 6 weeks in the presence of G418 $(400 \mu \mathrm{g} / \mathrm{ml})$. Control cells were transfected with empty vector. The expression of miR-144 in the stably transfected cells was evaluated by RT-qPCR. TAGLN shRNA Lentivirus (TAGLN-shRNA) and Negative Control shRNA Lentivirus (shRNA-NC) purchased from Santa Cruz Biotechnology, Inc. (Dallas, TX, USA) were used to tansfect the HOS and U2OS cells following the manufacturer's instructions and stably tansfected cells were selected by puromycin (Sigma, St. Louis, MO, USA).

Cell growth and invasion assay. The in vitro proliferation of the HOS and U2OS cells transfected with miR-NC, miR-144 mimics, TAGLN-shRNA or shRNA-NC was measured by 3-(4,5-dimethythiazol-2-yl)-2,5-diphenyl tetrazolium bromide (MTT) assay as previously described (12). Briefly, the cells were seeded onto 96-well plates and at the indicated periods to time, $100 \mu \mathrm{l}$ of medium was removed and replaced with fresh medium containing $0.5 \mathrm{mg} / \mathrm{ml}$ MTT solution. The plates were incubated at $37^{\circ} \mathrm{C}$ for $4 \mathrm{~h}$ and then the medium was replaced by $100 \mu \mathrm{l} \mathrm{DMSO}$ and the plates were shaken at room temperature for $10 \mathrm{~min}$. Absorbance was measured at $570 \mathrm{~nm}$.

The invasive potential of the cells was evaluated using Transwell inserts with $8 \mathrm{~mm}$ pores (Corning Inc., Corning, NY, USA). For invasion assay, at $24 \mathrm{~h}$ after transfection, $3.0 \times 10^{5}$ cells in serum-free medium were added to each upper insert precoated with Matrigel matrix (BD Biosciences, Bedford, MA, USA). Approximately $500 \mathrm{ml}$ of $10 \%$ FBS medium was added to the matched lower chamber. After $48 \mathrm{~h}$ of incubation, the non-invading cells were removed from the upper surface of the Transwell membrane with a cotton swab, and the invading cells on the lower membrane surface were fixed in methanol, stained with $0.1 \%$ crystal violet, photographed and counted. Six random fields at x100 magnification for each insert were counted. The inserts were analyzed in triplicate in 3 separate experiments.

3' Untranslated region (3' UTR) luciferase reporter assay. The micro-RNA database (www.microRNA.org) was used to find the putative miR-144 binding site in the 3' UTR of TAGLN (433 bp). The psiCHECK-2-TAGLN-3'UTR-wt vector or the psiCHECK-2-TAGLN-3'UTR-mut vector were co-transfected with control, miR-NC or miR-144 into the HOS cells using Lipofectamine 2000 (Invitrogen). Subsequently, reporter gene assays were performed 48 -h post-transfection using the Dual Luciferase Reporter assay system (Promega, Madison, WI, USA) according to the manufacturer's instructions. The normalized firefly luciferase activity was obtained by measuring firefly luciferase activity/Renilla luciferase activity. All experiments were performed at least 3 times.

Tumorigenicity assay in nude mice. A total of 54 BALB/c athymic nude mice were purchased from Shanghai SLAC Laboratory Animal Co., Ltd. (Shanghai, China). All animal protocols and procedures were approved by the Institutional Animal Care Utilization Committee, and were in accordance with the Johns Hopkins Animal Care and Use Committee guidelines. HOS cells stably transfected with miR-144 mimics, negative control (NC) RNA and control $\left(1 \times 10^{6}\right)$ were suspended in $0.1 \mathrm{ml}$ PBS and then injected subcutaneously into either side 

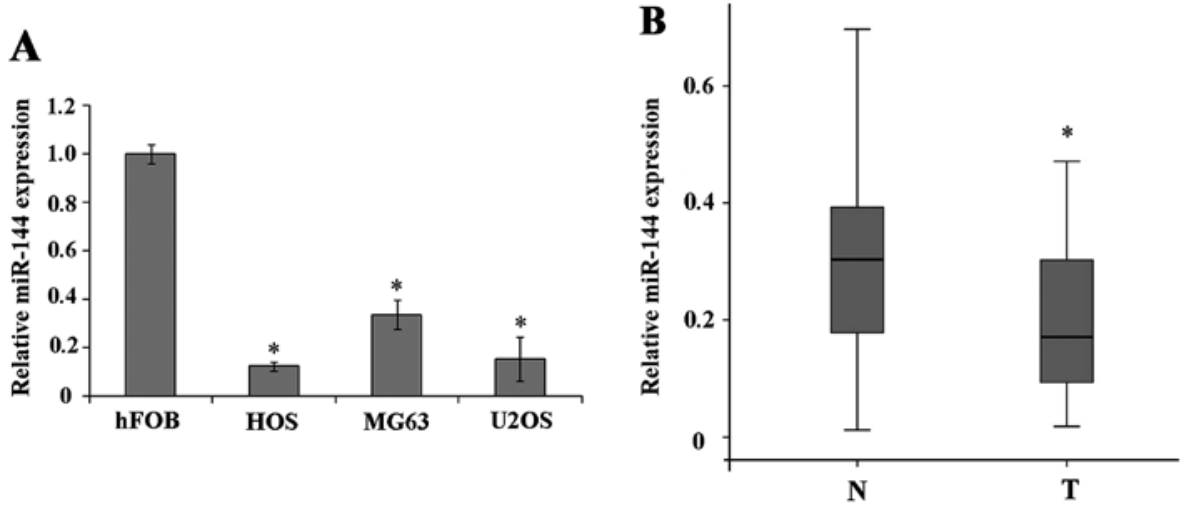

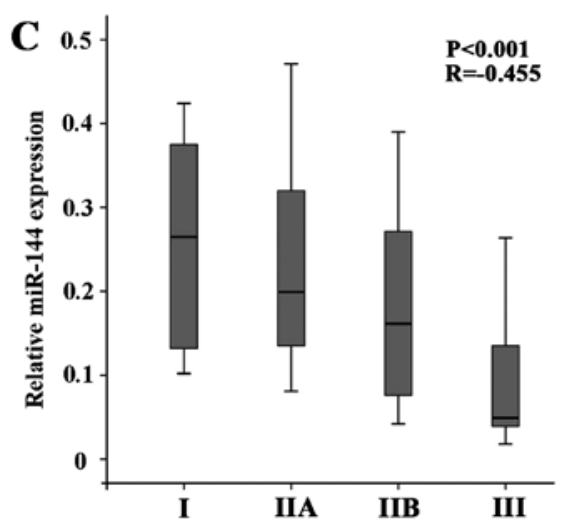

Figure 1. miR-144 is downregulated in osteosarcoma cell lines and clinical human osteosarcoma tissue samples. (A) miR-144 is downregulated in osteosarcoma cell lines (MG63, U2OS and HOS) in comparison with a control osteoblast cell line (hFOB1.19; hFOB). (B) miR-144 is downregulated in human osteosarcoma tissue samples (T) in comparison with paired adjacent normal tissue samples (N). The expression of miR-144 was normalized to that of U6 in each sample. Data are the means $\pm \mathrm{SD}(\mathrm{n}=4)$ in cell lines $(\mathrm{A})$ or as the horizontal lines (median), the boxes (interquartile range), and the whisker plots (2.5th and 97.5th percentiles) in human samples. (C) miR-144 downregulation correlates with the progression of osteosarcoma. In the 60 human osteosarcoma tissues, the correlation between miR-144 expression and tumor stages I to III was analyzed by Spearman's rank correlation assay with SPSS 17.0 software; the R and $\mathrm{P}$-values are indicated. Data are shown as in (C) of miR-144 expression in each stage. ${ }^{*} \mathrm{P}<0.05$.

of the posterior flank of the same BALB/c athymic nude mice at 4 weeks of age. Tumor growth was measured using calipers weekly, and tumor volume was calculated according to the following formula: volume $=$ length $\mathrm{x}$ width ${ }^{2} \times 0.5$.

Western blot analysis. Lysates obtained from the cells and tissue samples were extracted using the M-PER Protein Extraction Reagent (Pierce, Rockford, IL, USA), and the protein content was quantified using the BCA assay (Pierce). Equal amounts of extracts were subjected to SDS-PAGE, transferred onto polyvyniledene difluoride membranes and probed with primary antibodies against TAGLN (Sigma), matrix metalloproteinase 2 (MMP2; Abcam, Cambridge, MA, USA) and $\beta$-actin followed by the appropriate secondary antibodies (Cell Signaling Technology). Densitometric analysis was performed using LabWorks image acquisition and analysis software (UVP, Upland, CA, USA).

Statistical analysis. Data are presented as the means \pm SD. Statistical comparisons between groups were analyzed using a two-tailed Student's t-test and a P-value $<0.05$ was considered to indicate a statistically significant difference. The correlation between miR-144 expression and clinical osteosarcoma stages was analyzed using the Spearman's rank correlation assay with SPSS 17.0 software (SPSS Inc., Chicago, IL, USA). The correlation between miR-144 expression and TAGLN mRNA levels was analyzed using the Spearman's correlation coefficient assay with SPSS 17.0 software.

\section{Results}

miR-144 is downregulated in osteosarcoma. To determine the role of miR-144 in the development of human osteosarcoma, we analyzed miR-144 expression in the human osteosarcoma cell lines, MG63, U2OS and HOS, by RT-qPCR and compared it to that in the normal human osteoblastic cell line, hFOB1.19. The results revealed that miR-144 was significantly downregulated in the osteosarcoma cell lines compared to the normal osteoblastic cells (Fig. 1A; $\mathrm{P}<0.05)$. We then analyzed miR-144 expression in the paired human osteosarcoma tumor tissue and adjacent normal tissue samples; the results revealed a significant downregulation of miR-144 expression in tumor tissues compared to adjacent normal tissues (Fig. 1B; $\mathrm{P}<0.05$ ). To determine whether the downregulation of miR-144 is correlated with the progression and development of osteosarcoma, we used Spearman's rank correlation, which revealed a significant negative correlation between miR-144 expression in tumor tissue and clinical stage (Fig. 1C). These results suggest that the downregulation of miR-144 expression plays a role in the progression of osteosarcoma.

miR-144 inhibits osteosarcoma cell growth and invasion. To further investigate the biological role of miR-144 in osteosarcoma tumor progression, the HOS and U2OS cell lines were infected with lentivirus carrying a negative control miR (miR-NC) or miR-144 mimics and miR-144 expression was analyzed by RT-qPCR $72 \mathrm{~h}$ after infection. The results revealed that miR-144 expression was significantly upregulated in the miR-144-infected cells compared to the cells infected with miR-NC (Fig. 2A) $(\mathrm{P}<0.01)$. To determine the effects of miR-144 on osteosarcoma cell growth, the viability of the HOS and U2OS cells treated as described above was assessed by MTT assay at the indicated time points. The results revealed that transfection with miR-144 mimics significantly decreased the growth of osteosarcoma cells compared to the control- or miR-NC-transfected cells (Fig. 2B; $\mathrm{P}<0.05$ at 72 and $96 \mathrm{~h}$ ). Furthermore, the assessment of cell invasion by Transwell assay revealed a significant impairment in the invasive ability of the cells expressing miR-144 mimics compared to that of the control- or miR-NC-transfected cells (Fig. 2C; P<0.05).

TAGLN expression is upregulated in osteosarcoma and correlates with miR-144 expression. To examine the correlation between miR-144 and TAGLN in osteosarcoma, the expression 

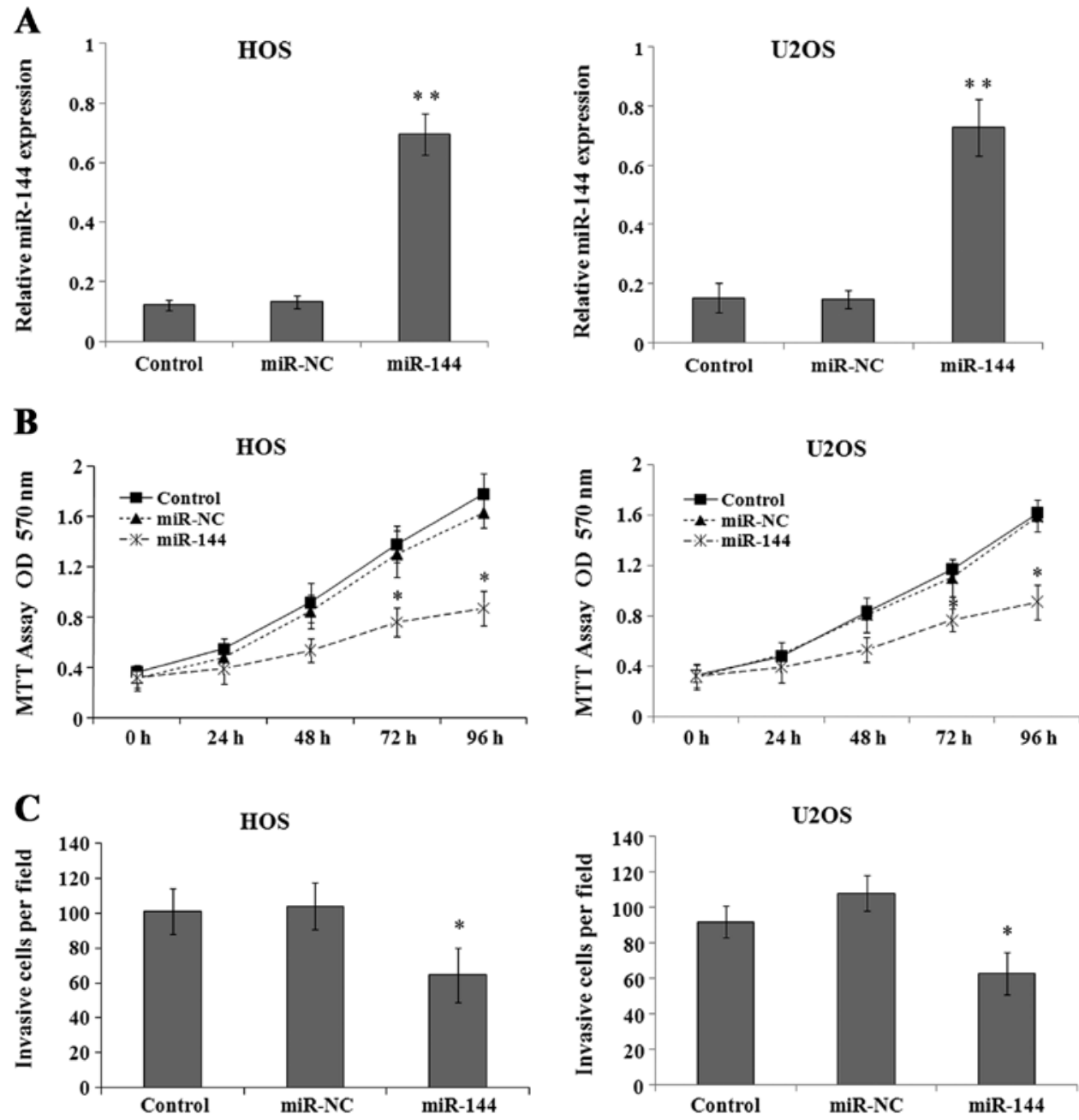

Figure 2. miR-144 suppresses osteosarcoma cell migration and invasion. (A) miR-144 expression levels were upregulated significantly in the HOS and U2OS cells transfected with miR-144 compared with the control and miR-NC-transfected cells, as shown by RT-qPCR. (B) Osteosarcoma HOS and U2OS cells were transfected with control RNA or miR-144 mimics as indicated. Cell proliferation was measured at the indicated time periods of time post transfection by MTT assay. (C) Upregulation of miR-144 significantly inhibited cell invasion as quantified by cell counting. Similar results were obtained in 3 independent experiments. ${ }^{*} \mathrm{P}<0.05,{ }^{* *} \mathrm{P}<0.01$.
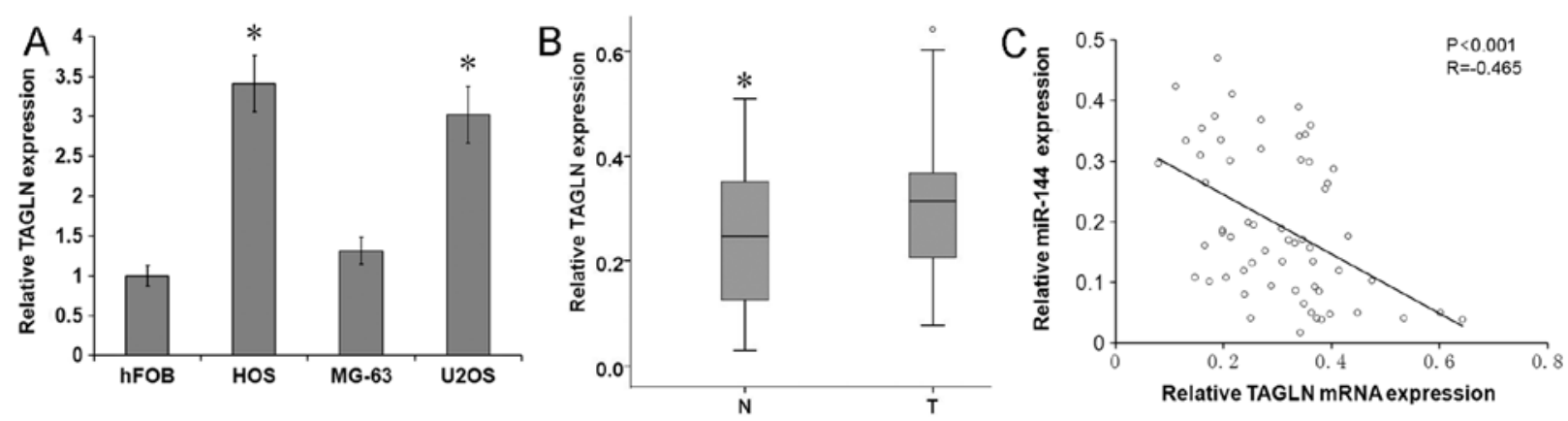

Figure 3. TAGLN expression is upregulated in osteosarcoma. (A) TAGLN expression was upregulated in osteosarcoma cell lines (MG63, U2OS and HOS) compared with a control osteoblast cell line (hFOB1.19; hFOB), as determined by RT-qPCR. (B) TAGLN was upregulated in human osteosarcoma tissues in comparison with paired adjacent normal tissues. (C) Spearman's correlation analysis was used to determine the correlation between the expression levels of TAGLN and miR-144 in osteosarcoma tissues by SPSS software (O, expression of miR-144 and TAGLN in normal bone and myeloid tissue samples and osteosarcoma tissue samples relative to zero) (Spearman's correlation analysis, $\mathrm{R}=-0.465 ; \mathrm{P}<0.001)$. Data are presented as in $(\mathrm{C})$ of TAGLN expression and as in Fig. 1C of miR-144 expression at each clinical stage. ${ }^{*} \mathrm{P}<0.05$.

of TAGLN was analyzed by RT-qPCR in the osteosarcoma cell lines and primary tumor tissue samples. The results revealed that the TAGLN mRNA level was significantly higher in the osteosarcoma cell lines, U2OS and HOS, than in the control human osteoblast cell line, hFOB1.19 (Fig. 3A; P<0.05), and it was significantly higher in the human primary osteosarcoma 
A

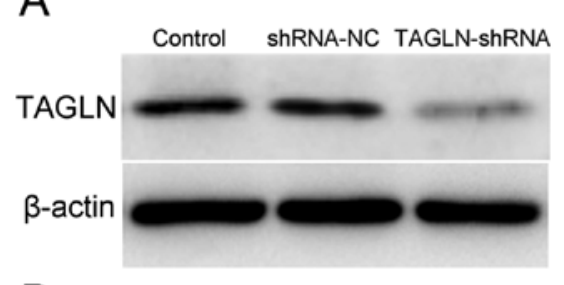

B

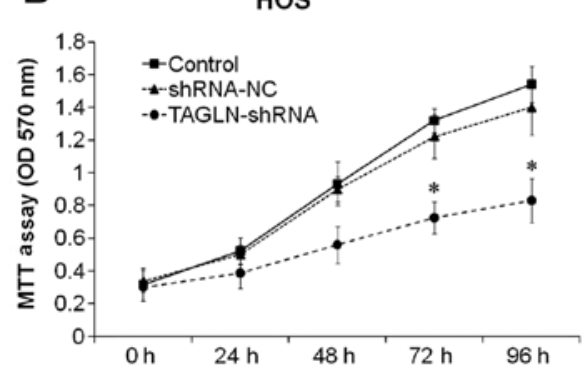

C

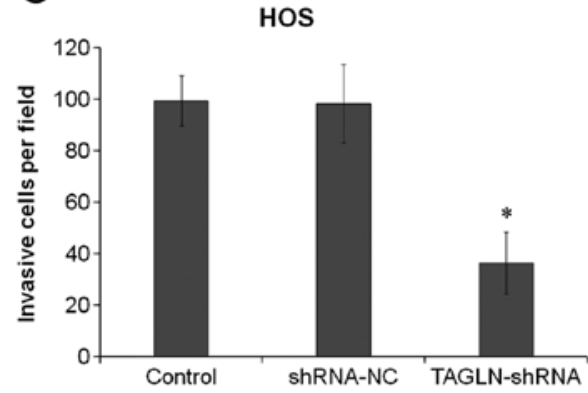

U2OS

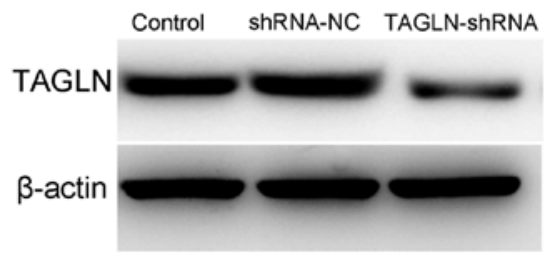

U2OS

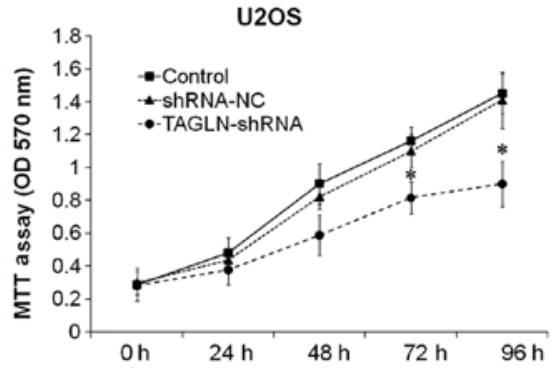

U2OS

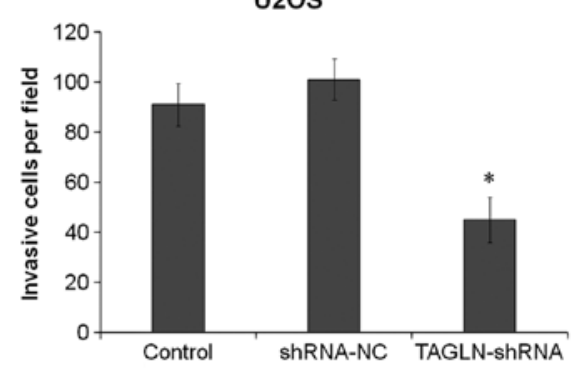

Figure 4. Effect of TAGLN-siRNA on the growth and invasion of osteosarcoma cells. (A) HOS and U2OS cells were transfected with a TAGLN-siRNA or control-shRNA. TAGLN protein levels were quantified by western blot analysis. The expression of TAGLN was decreased in the cells transfected with TAGLN-siRNA when compared with the cells transfected with shRNA-NC and the control. (B) Downregulation of TAGLN expression in HOS and U2OS cells suppressed cell growth. (C) Downregulation of TAGLN expression decreased the invasive capacity of HOS and U2OS cells. *P<0.05.

tumor tissue samples than in the adjacent normal tissue samples (Fig. 3B; P<0.05). To identify a potential correlation between the expression of miR-144 and TAGLN in osteosarcoma tissue, we used Spearman's correlation, which revealed a significant inverse correlation between miR-144 and TAGLN mRNA expression (Fig. 3C; $\mathrm{R}=-0.465, \mathrm{P}<0.001$ ), confirming that the downregulation of miR-144 is associated with the upregulation of TAGLN in osteosarcoma.

TAGLN silencing inhibits osteosarcoma cell growth and invasion. To further determine whether TAGLN is the primary regulator of cell invasion and migration in osteosarcoma, TAGLN was stably silenced in the HOS and U2OS cells. The cells were transfected with TAGLN-shRNA and stable transfectants were obtained after $48 \mathrm{~h}$. The expression of TAGLN was determined by western blot analysis, which revealed that the protein levels of TAGLN were significantly decreased in the HOS and U2OS cells (both $\mathrm{P}<0.05$ ). HOS and U2OS cells stably depleted of TAGLN were cultured and cell viability and invasion were assessed by MTT and Transwell assays. As shown in Fig. 4B, the depletion of TAGLN significantly $(\mathrm{P}<0.05)$ reduced the viability of the HOS and U2OS cells compared with the respective negative control shRNA-transfected cells. The results of Transwell assay revealed that the silencing of TAGLN significantly inhibited the invasive ability of the HOS and U2OS cells compared to that of the control- or negative control shRNA-transfected cells (Fig. 4C; $\mathrm{P}<0.05$ ).

miR-144 directly targets TAGLN. Potential miR-144 binding sites in the 3' UTR of TAGLN were predicted using microRNA.org (www.microRNA.org). A sequential replacement of a 5 base pair region was performed to produce a mutant vector (Fig. 5A). To further investigate whether the predicted binding site of miR-144 in the 3' UTR of TAGLN is responsible for the interaction, the 3' UTR of TAGLN was cloned downstream to a luciferase reporter gene (wt-TAGLN), and a mutant version (mut-TAGLN) was generated by binding site mutagenesis. The wt-TAGLN vector or mut-TAGLN with control, negative control or miR-144 mimics was cloned into the HOS cells and the luciferase activity was determined. The results revealed that the luciferase activity of the miR-144-transfected cells was significantly reduced compared with that of the negative control-transfected cells (Fig. 5B). To further confirm that TAGLN is a direct target of miR-144, the protein levels of TAGLN were assessed by western blot anlaysis in the cells transfected with miR-144 mimics, which indicated that the exogenous expression of miR-144 significantly downregulated TAGLN expression in the HOS and U2OS cells (Fig. 5C). 
$\mathbf{A}$

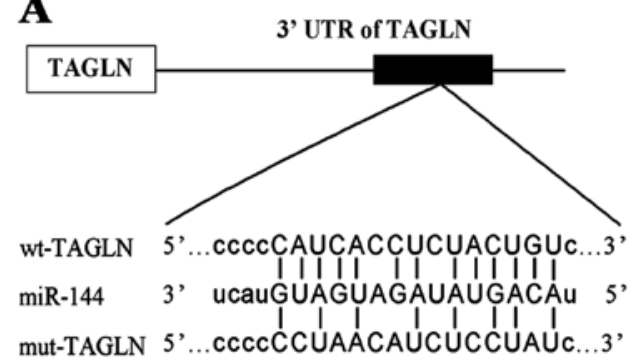

\section{B}

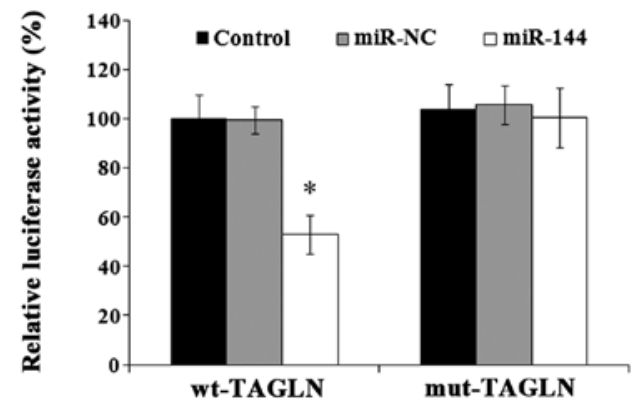

$\mathbf{C}$
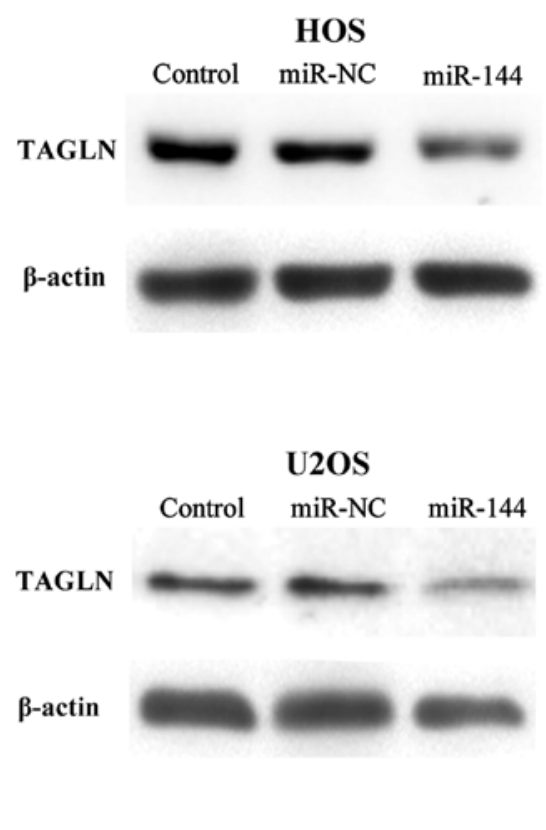

Figure 5. TAGLN is a direct target of miR-144. (A) Sequence alignment of miR-144 and its predicted target site in the 3' UTR of TAGLN are shown as indicated. Data were downloaded from microRNA.org (http://www.microrna.org). (B) HOS cells were co-transfected with a TAGLN 3 ' UTR firefly luciferase reporter plasmid and Renilla plasmids, control RNA or miR-144 mimics as indicated. After 48 h, firefly luciferase activity was measured and normalized to Renilla luciferase activity as indicated and calculated as the ratio of firefly/Renilla activities in the cells and normalized to those of the control. The results are presented as the means \pm SD from 3 independent experiments with each experiment performed in triplicate. (C) TAGLN protein expression was decreased by miR-144 when compared with that of the cells transfected with miR-NC and the control, as shown by western blot analysis.
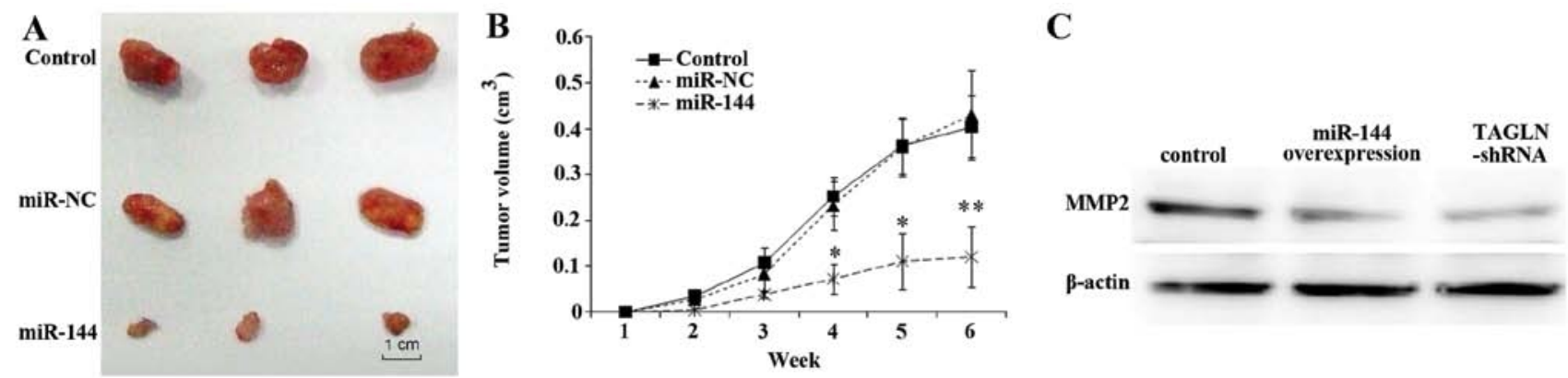

Figure 6. Effect of miR-144 overexpression on tumorigenicity. (A) miR-144 attenuated tumor growth in nude mice (the tumors were obtained at 6 weeks after subcutaneous injection). (B) Tumor growth was measured and volume curves were drawn as indicated. Data are expressed as the means \pm SD (n=4) or presented separately for one representative experiment. Similar results were obtained in 3 independent experiments. (C) MMP-2 expression was downregulated in the TAGLN-siRNA-transfected group or in the miR-144-transfected group compared with the control. ${ }^{*} \mathrm{P}<0.05,{ }^{* *} \mathrm{P}<0.01$

miR-144 overexpression inhibits tumorigenicity. The osteosarcoma cell line, HOS, was stably transfected with miR-144 mimics and negative control (NC) RNA, as previously described (18) and implanted subcutaneously into nude mice to examine tumorigenicity in vivo. The effects were most prominent in the first week after inoculation, in which a significant suppression of tumorigenicity by miR-144 was observed as determined by the significantly smaller tumors formed in the mice (Fig. 6A). Fig. 6B shows the reduction in tumor volume in mice carrying miR-144-overexpressing tumors compared to the control mice or mice transplanted with negative control-transfected cells. To confirm the effects of miR-144 and its target, TAGLN, on invasion in vivo, the expression of proteins involved in cell adhesion and migration was determined. The results revealed that the expression of MMP2 was downregulated by TAGLN-siRNA or by miR-144 overexpression (Fig. 6C). Taken together, these results indicate that miR-144 functions as a tumor suppressor in osteosarcoma through the modulation of its target gene, TAGLN.

\section{Discussion}

Osteosarcoma is the most common human primary malignant bone tumor and is associated with an aggressive clinical course. In recent years, the investigation of the signaling pathways that regulate osteosarcoma carcinogenesis, invasion and metastasis has gained increasing attention $(19,20)$, in particular the identification of deregulated miRNAs and their role in the 
development of osteosarcoma (12-14). Recent accumulating evidence suggests that the aberrant expression of miRNAs in cancer is not a random event, but rather plays an important role in tumorigenic processes $(6,10)$. Studies on tumor invasion and metastasis have revealed the critical role of miRNAs (7,21-24) in these processes, and have provided potential therapeutic targets for anti-metastatic therapies.

The TAGLN gene codes for transgelin, an actin stress fiber binding protein that plays a role in cell growth, differentiation, migration invasion and matrix remodeling by stabilizing the cytoskeleton through actin binding (25-27). Previous studies have demonstrated that the expression of TAGLN is significantly reduced in tissues of the urinary bladder, renal cell carcinoma and colorectal carcinoma compared to matched normal tissues (28-30). In colorectal carcinoma, the overexpression of TAGLN has been shown to decrease proliferation and invasion (29), supporting a role for transgelin as a tumor suppressor. However, the increased expression of transgelin has been reported in colorectal adenocarcinoma, prostate carcinoma, hepatocellular carcinoma, gastric, pancreatic and colon cancer (26,31-35). Elevated levels of transgelin have been shown to significantly increase the invasiveness of tumor cells (26), whereas reduced transgelin expression markedly interferes with invasiveness (36). In gastric carcinoma, the upregulation of TAGLN expression promotes tumor metastasis, and the effects of increased TAGLN expression on enhancing tumor cell invasion and migration are mediated by the upregulation of the expression of MMP2 (37). Previous studies have shown that MMP2 regulates cell migration and invasion in cancer and its activity is, therefore, linked to the process of metastasis (38-40).

In the present study, we hypothesized that miR-144 may be involved in the metastatic process in osteosarcoma. We first identified TAGLN as a direct target of miR-144 and confirmed their interaction. We then demonstrated that miR-144 inhibited osteosarcoma cell invasion by targeting TAGLN. Our RT-qPCR results revealed that miR-144 was downregulated in osteosarcoma cell lines, which was consistent with the results obtained in 60 osteosarcoma tissue samples. Conversely, we showed that TAGLN was upregulated in osteosarcoma cell lines and tissue samples. The exogenous expression of miR-144 or the knockdown of TAGLN expression in the HOS and U2OS cells resulted in the inhibition of cell growth and invasion as determined by MTT and Transwell assays. Furthermore, miR-144 levels negatively correlated with the TAGLN mRNA levels in osteosarcoma tissue samples, confirming the importance of the molecular association between miR-144 and TAGLN. The results of a luciferase reporter assay confirmed that miR-144 directly targets the TAGLN gene by binding to a specific complementary site within its 3 ' UTR, resulting in the suppression of TAGLN expression by miR-144. Furthermore, the expression of MMP2 was downregulated by TAGLN-siRNA or by miR-144 overexpression. Despite our findings showing an association between miR-144 and TAGLN and their effects on the expression of invasion-related molecules, further investigation is required to elucidate the specific mechanisms underlying their effects on the metastatic potential of osteosarcoma cells. Nevertheless, our findings provide clear evidence that miR-144 plays a tumor suppressive role, inhibiting cellular proliferation and invasion, at least in part through the downregulation of TAGLN expression.
In the present study, we identified TAGLN as a direct target of miR-144 in osteosarcoma, suggesting that miR-144 exerts its anti-metastatic effects by inhibiting TAGLN expression. Previous studies have indicated that human zinc finger protein, X-linked (ZFX), enhancer of zeste homolog 2 (EZH2) and phosphatase and tensin homolog (PTEN) are molecular targets of miR-144 and they all participate in cancer development (41-44). Our data suggest that the anti-metastatic effects of miR-144 in osteosarcoma cells are mediated mainly through the inhibition of its target, TAGLN. However, a single miRNA can target multiple mRNAs to modulate gene expression (45). Hence, other miR-144 targets may exist that have not yet been identified, and their effects on osteosarcoma carcinogenesis and progression remain to be elucidated. Future studies are required to identify the targetome and roles of miR-144 in cancer development.

In conclusion, to the best of our knowledge, we describe for the first time a miR-144/TAGLN link and provide a potential mechanism for TAGLN deregulation and its contribution to osteosarcoma cell proliferation and invasion. Further studies are required focusing on elucidating the precise regulatory mechanisms of miR-144 and its contribution to the proliferation and invasion of osteosarcoma cells.

\section{Acknowledgements}

The present study was supported by grants from the Shanghai Municipal Health Bureau youth Foundation (2012-138, 2012-167), the Nature Science Foundation (81202160), the Shanghai Municipal Health Bureau Key Project (2011-19) and the Shanghai Young Physician Training Program (2012-30, 2012-31).

\section{References}

1. Bielack SS, Kempf-Bielack B, Delling G, et al: Prognostic factors in high-grade osteosarcoma of the extremities or trunk: an analysis of 1,702 patients treated on neoadjuvant cooperative osteosarcoma study group protocols. J Clin Oncol 20: 776-790, 2002.

2. Marina N, Gebhardt M, Teot L and Gorlick R: Biology and therapeutic advances for pediatric osteosarcoma. Oncologist 9: 422-441, 2004

3. Bartel DP: MicroRNAs: genomics, biogenesis, mechanism, and function. Cell 116: 281-297, 2004.

4. Bushati $\mathrm{N}$ and Cohen SM: microRNA functions. Annu Rev Cell Dev Biol 23: 175-205, 2007.

5. Griffiths-Jones S: miRBase: the microRNA sequence database. Methods Mol Biol 342: 129-138, 2006.

6. Hwang HW and Mendell JT: MicroRNAs in cell proliferation, cell death, and tumorigenesis. Br J Cancer 94: 776-780, 2006

7. Yan K, Gao J, Yang T, et al: MicroRNA-34a inhibits the proliferation and metastasis of osteosarcoma cells both in vitro and in vivo. PloS One 7: e33778, 2012.

8. Calin GA and Croce CM: MicroRNA signatures in human cancers. Nat Rev Cancer 6: 857-866, 2006.

9. Kumar MS, Lu J, Mercer KL, Golub TR and Jacks T: Impaired microRNA processing enhances cellular transformation and tumorigenesis. Nat Genet 39: 673-677, 2007.

10. Zhang B, Pan X, Cobb GP and Anderson TA: microRNAs as oncogenes and tumor suppressors. Dev Biol 302: 1-12, 2007.

11. Kobayashi E, Hornicek FJ and Duan Z: MicroRNA involvement in osteosarcoma. Sarcoma 2012: 35973, 2012.

12. Zhang H, Cai X, Wang Y, Tang H, Tong D and Ji F: microRNA-143, down-regulated in osteosarcoma, promotes apoptosis and suppresses tumorigenicity by targeting Bcl-2. Oncol Rep 24: 1363-1369, 2010

13. Ji F, Zhang H, Wang Y, et al: MicroRNA-133a, downregulated in osteosarcoma, suppresses proliferation and promotes apoptosis by targeting Bcl-xL and Mcl-1. Bone 56: 220-226, 2013. 
14. Jin Y, Peng D, Shen Y, et al: MicroRNA-376c inhibits cell proliferation and invasion in osteosarcoma by targeting to transforming growth factor-alpha. DNA Cell Biol 32: 302-309, 2013.

15. Namlos HM, Meza-Zepeda LA, Baroy T, et al: Modulation of the osteosarcoma expression phenotype by microRNAs. PloS One 7: e48086, 2012.

16. Sureban SM, May R, Mondalek FG, et al: Nanoparticle-based delivery of siDCAMKL-1 increases microRNA-144 and inhibits colorectal cancer tumor growth via a Notch-1 dependent mechanism. J Nanobiotechnology 9: 40, 2011.

17. Livak KJ and Schmittgen TD: Analysis of relative gene expression data using real-time quantitative PCR and the $2^{-\Delta \Delta C T}$ Method. Methods 25: 402-408, 2001.

18. Diaz-Prado S, Cicione C, Muinos-LopezE, et al: Characterization of microRNA expression profiles in normal and osteoarthritic human chondrocytes. BMC Musculoskelet Disord 13: 144, 2012.

19. Guo YS, Zhao R, Ma J, et al: $\beta$ ig-h3 promotes human osteosarcoma cells metastasis by interacting with integrin $\alpha 2 \beta 1$ and activating PI3K signaling pathway. PloS One 9: e90220, 2014.

20. Lin CH, Guo Y, Ghaffar S, et al: Dkk-3, a secreted wnt antagonist, suppresses tumorigenic potential and pulmonary metastasis in osteosarcoma. Sarcoma 2013: 147541, 2013.

21. Duan Z, Choy E, Harmon D, et al: MicroRNA-199a-3p is downregulated in human osteosarcoma and regulates cell proliferation and migration. Mol Cancer Ther 10: 1337-1345, 2011.

22. Fan L, Wu Q, Xing X, Wei Y and Shao Z: MicroRNA-145 targets vascular endothelial growth factor and inhibits invasion and metastasis of osteosarcoma cells. Acta Biochim Biophys Sin 44: 407-414, 2012

23. Bao YP, Yi Y, Peng LL, et al: Roles of microRNA-206 in osteosarcoma pathogenesis and progression. Asian Pac J Cancer Prev 14: 3751-3755, 2013.

24. Jin J, Cai L, Liu ZM and Zhou XS: miRNA-218 inhibits osteosarcoma cell migration and invasion by down-regulating of TIAM1, MMP2 and MMP9. Asian Pac J Cancer Prev 14: 3681-3684, 2013

25. Yang Z, Chang YJ, Miyamoto H, et al: Transgelin functions as a suppressor via inhibition of ARA54-enhanced androgen receptor transactivation and prostate cancer cell growth. Mol Endocrinol 21: 343-358, 2007

26. Lee EK, Han GY, Park HW, Song YJ and Kim CW: Transgelin promotes migration and invasion of cancer stem cells. J Proteome Res 9: 5108-5117, 2010.

27. Thompson O, Moghraby JS, Ayscough KR and Winder SJ: Depletion of the actin bundling protein SM22/transgelin increases actin dynamics and enhances the tumourigenic phenotypes of cells. BMC Cell Biol 13: 1, 2012.

28. Assinder SJ, Stanton JA and Prasad PD: Transgelin: an actinbinding protein and tumour suppressor. Int J Biochem Cell Biol 41: 482-486, 2009.

29. Li Q, Shi R, Wang Y and Niu X: TAGLN suppresses proliferation and invasion, and induces apoptosis of colorectal carcinoma cells. Tumour Biol 34: 505-513, 2013.

30. Chunhua L, Donglan L, Xiuqiong F, et al: Apigenin up-regulates transgelin and inhibits invasion and migration of colorectal cancer through decreased phosphorylation of AKT. J Nutr Biochem 24: 1766-1775, 2013.
31. Lin Y, Buckhaults PJ, Lee JR, et al: Association of the actinbinding protein transgelin with lymph node metastasis in human colorectal cancer. Neoplasia 11: 864-873, 2009.

32. Shi YY, Wang HC, Yin YH, et al: Identification and analysis of tumour-associated antigens in hepatocellular carcinoma. Br J Cancer 92: 929-934, 2005.

33. Li N, Zhang J, Liang Y, et al: A controversial tumor marker: is SM22 a proper biomarker for gastric cancer cells? J Proteome Res 6: 3304-3312, 2007.

34. Huang Q, Huang Q, Chen W, et al: Identification of transgelin as a potential novel biomarker for gastric adenocarcinoma based on proteomics technology. J Cancer Res Clin Oncol 134: 1219-1227, 2008.

35. Mikuriya K, Kuramitsu Y, Ryozawa S, et al: Expression of glycolytic enzymes is increased in pancreatic cancerous tissues as evidenced by proteomic profiling by two-dimensional electrophoresis and liquid chromatography-mass spectrometry/mass spectrometry. Int J Oncol 30: 849-855, 2007.

36. $\mathrm{Yu} \mathrm{H}$, Konigshoff M, Jayachandran A, et al: Transgelin is a direct target of TGF-beta/Smad3-dependent epithelial cell migration in lung fibrosis. FASEB J 22: 1778-1789, 2008.

37. Yu B, Chen X, Li J, et al: Stromal fibroblasts in the microenvironment of gastric carcinomas promote tumor metastasis via upregulating TAGLN expression. BMC Cell Biol 14: 17, 2013.

38. Wang $\mathrm{H}$, Zhu Y, Zhao M, et al: miRNA-29c suppresses lung cancer cell adhesion to extracellular matrix and metastasis by targeting integrin $\beta 1$ and matrix metalloproteinase2 (MMP2). PloS One 8: e70192, 2013.

39. Wang XX, Cheng Q, Zhang SN, et al: PAK5-Egr1-MMP2 signaling controls the migration and invasion in breast cancer cell. Tumour Biol 34: 2721-2729, 2013.

40. Yu G, Li H, Wang X, et al: MicroRNA-19a targets tissue factor to inhibit colon cancer cells migration and invasion. Mol Cell Biochem 380: 239-247, 2013

41. Akiyoshi S, Fukagawa $\mathrm{T}$, Ueo $\mathrm{H}$, et al: Clinical significance of miR-144-ZFX axis in disseminated tumour cells in bone marrow in gastric cancer cases. Br J Cancer 107: 1345-1353, 2012.

42. Zha W, Cao L, Shen Y and Huang M: Roles of Mir-144-ZFX pathway in growth regulation of non-small-cell lung cancer. PloS One 8: e74175, 2013

43. Guo Y, Ying L, Tian Y, et al: miR-144 downregulation increases bladder cancer cell proliferation by targeting EZH2 and regulating Wnt signaling. FEBS J 280: 4531-4538, 2013.

44. Zhang LY, Ho-Fun Lee V, Wong AM, et al: MicroRNA-144 promotes cell proliferation, migration and invasion in nasopharyngeal carcinoma through repression of PTEN. Carcinogenesis 34: 454-463, 2013.

45. Selbach M, Schwanhausser B, Thierfelder N, Fang Z, Khanin R and Rajewsky N: Widespread changes in protein synthesis induced by microRNAs. Nature 455: 58-63, 2008. 